\title{
Decitabine in the treatment of acute myeloid leukemia in elderly patients
}

\author{
This article was published in the following Dove Press journal: \\ Cancer Management and Research \\ 3 February 2014 \\ Number of times this article has been viewed
}

\author{
Priya Malik \\ Amanda F Cashen \\ Washington University School of \\ Medicine, St Louis, MO, USA
}

Correspondence: Amanda F Cashen

Washington University School of Medicine, 660 South Euclid Ave,

St Louis, MO 63II0, USA

Tel + I 3|4 4548306

Fax + I 3 |4 454755 I

Email acashen@dom.wustl.edu

\begin{abstract}
The majority of patients with acute myeloid leukemia (AML) are elderly and have a poor prognosis despite induction therapy. Decitabine, a DNA-hypomethylating agent that induces differentiation and apoptosis of leukemic cells, is a well-tolerated alternative to aggressive chemotherapy. It is currently FDA-approved for myelodysplastic syndrome, including patients with $20 \%-30 \%$ bone marrow blasts. Recent clinical attention has focused on evaluating decitabine as frontline therapy for untreated high-risk elderly AML patients. A large randomized international phase III study comparing decitabine to supportive care and cytarabine in elderly AML patients demonstrated significantly improved complete remission rates, but the survival difference did not reach significance. Due to this, decitabine did not achieve FDA approval for AML, but continues to be used off-label. Current research is focused on further defining subgroups of elderly AML patients who may derive greater benefit from decitabine therapy and combining it with other low-intensity active agents for AML.
\end{abstract}

Keywords: decitabine, elderly, AML

\section{Introduction}

The majority of the approximately 14,500 individuals diagnosed with acute myeloid leukemia (AML) each year in the US are over age 60 years, and a third are over age 75 years. ${ }^{1}$ Unfortunately, advanced age is one of the most adverse prognostic factors in AML, in part because older patients have difficulty tolerating chemotherapy due to comorbidities, concomitant end-organ dysfunction, and poor performance status. However, the biology of the disease in the elderly also contributes to poor outcomes. AML in older patients can be secondary to overt or unrecognized myelodysplastic syndromes (MDS), which tends to be less chemoresponsive. AML in the elderly is also associated with complex and monosomal karyotypes with adverse cytogenetics, ${ }^{2-6}$ fewer favorable mutations such as $N P M 1,{ }^{5}$ and a multidrug-resistant phenotype. ${ }^{6}$ Even within each molecular risk group, older patients tend to have a more chemoresistant disease, the precise mechanisms for which are unknown.

Due to the high morbidity and relatively low efficacy associated with standard induction chemotherapy for elderly patients, there has been considerable interest in the hypomethylating agents 5-azacitadine and decitabine as frontline therapy. Both drugs were originally developed as cytarabine analogues to be used at high doses as antimetabolites, until their ability to target epigenetic changes that contribute to leukemogenesis was discovered. While their precise mechanism of action is unclear, it is thought that by reversing methylation-induced gene expression and perhaps through direct cytotoxicity, both agents can reactivate tumor-suppressor genes and promote apoptosis. ${ }^{7.8}$ 
They are currently FDA-approved for MDS, after trials demonstrated complete and partial remissions lasting several months when compared to supportive therapy, with a tolerable toxicity profile. ${ }^{9-11}$ This article addresses the clinical evidence, toxicity, and potential role of decitabine in previously untreated elderly patients with AML.

\section{Treatment options for elderly AML patients}

There are several distinct therapy pathways for elderly adults diagnosed with AML. For patients age 60-65 years with good performance status and favorable cytogenetics, induction with the $7+3$ regimen of cytarabine and an anthracycline (daunorubicin, idarubicin, or mitoxantrone) offers the possibility of durable disease control and may be the best treatment option. In fact, a study by Löwenberg et al suggested that these patients may benefit from higher doses of anthracyclines ( $90 \mathrm{mg} / \mathrm{m}^{2} /$ day instead of $45 \mathrm{mg} / \mathrm{m}^{2} /$ day of daunorubicin) with reported complete remission (CR) rates of $64 \%$ versus $54 \%$. ${ }^{12}$ However, the benefits of the higher-dose anthracycline were limited to patients under 65 years old. Responses can be consolidated with additional cycles of cytarabine or reduced-intensity conditioning allogeneic stem cell transplantation.

While remission rates of $40 \%-50 \%$ with the $7+3$ regimen have been noted in elderly patients in clinical trials, median survival is still dismal at 7-12 months, with a 7\% 2-year survival in some subgroups. ${ }^{13-15}$ Induction chemotherapy is also associated with significant upfront morbidity, with 4-week mortality of $15 \%-30 \%$ in select elderly populations. ${ }^{13,14,16}$ In addition, many elderly patients are unable to undergo allogeneic stem cell transplantation due to poor performance status, comorbidities, and older sibling age, leading to transplant rates of $5 \%$ in one prospective feasibility study. ${ }^{17}$ For elderly patients with intermediate- or high-risk cytogenetics or unfavorable molecular markers who are not candidates for transplantation, the limited curative potential and high toxicity of standard chemotherapy has led many patients and oncologists to pursue alternative options. For many years, the primary option was supportive care and referral to a hospice. This path was chosen by over two-thirds of older adults with AML during the 1990s and was associated with a median survival of only 1 month. ${ }^{18}$

A number of intermediate-intensity therapy regimens have now been investigated for the large subset of elderly patients over 65 years old who are unlikely to benefit from aggressive induction therapy but who desire treatment. While not curative, these agents offer the promise of remission and/or stabilization of counts, allowing additional months of quality life outside the hospital. Low-dose cytarabine (LDAC) has been established as the prototype of this middle route after a randomized study by Burnett et al showed higher rates of CR (18\% versus 1\%) and overall survival compared to supportive care in a population of elderly adults who were not candidates for conventional therapy. ${ }^{19}$ However, overall survival was only 4 months for the LDAC cohort, a modest improvement over the 3 months seen in the hydroxyurea group. Additionally, for patients with adverse cytogenetics, no benefit in remission or survival was noted. Gemtuzumab ozogamicin had shown some promise in elderly patients, ${ }^{20}$ but it was withdrawn from the market in 2010 after a postmarketing confirmatory phase III trial was terminated for lack of definitive benefit and enhanced toxicity. ${ }^{21}$ Aside from clinical trials, the current National Comprehensive Cancer Care guidelines suggest clofarabine, 5-azacitidine, and decitabine as alterative options for this population of patients. ${ }^{22}$ Clofarabine ${ }^{23}$ and 5 -azacitadine ${ }^{24}$ have been reviewed elsewhere.

\section{Decitabine}

Decitabine (5-aza-2'-deoxycytidine or 5-Aza-Cdr) is a cytosine analogue that was first synthesized in the early $1960 \mathrm{~s}$ by Pliml and Sorm and is currently marketed as Dacogen ${ }^{\circledR}$ by Eisai (Tokyo, Japan). It differs from deoxycytidine by the substitution of nitrogen for carbon at the 5-position of the pyrimidine ring (Figure 1). It was noted to have an antileukemic effect in cell lines, with more potency in vitro than cytarabine. ${ }^{25}$ Initially, its cytotoxicity was attributed to its ability to impair DNA synthesis and cause DNA damage similar to other antimetabolites. Early clinical experience therefore utilized this drug in high doses (up to $1,000 \mathrm{mg} / \mathrm{m}^{2}$ per cycle) alone and in combination with anthracyclines, with considerable hematological and nonhematological toxicity. ${ }^{26,27}$

Preclinical studies in the 1980s, however, demonstrated that at low doses, decitabine induced differentiation by reversing DNA methylation-induced gene silencing. ${ }^{28}$ Once inside a cell, decitabine is phosphorylated and activated by the enzyme deoxycytidine kinase to its triphosphate form aza-dCTP. ${ }^{29,30}$ It then competes with and replaces cytosine in the $\mathrm{CpG}$ (cytosine-guanosine dinucleotide) islands that occur in clusters in promoter regions. During subsequent cell divisions, aza-dCTP inhibits methylation of the promoter by forming a covalent bond with the enzyme DNA methyltransferase (DNMT), and thereby traps and contributes to degradation of the enzyme. ${ }^{29,31}$ Since methylation-induced aberrant transcriptional silencing of tumor-suppressor genes has been 


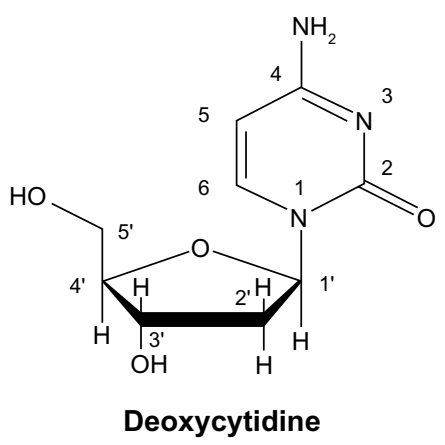

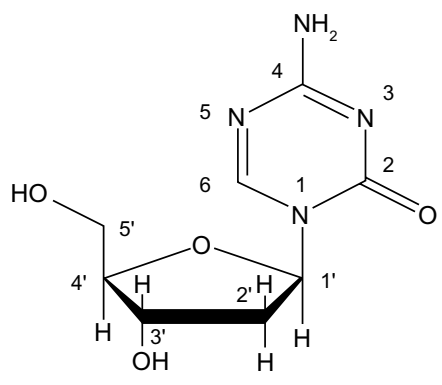

5-aza-2'-deoxycytidine

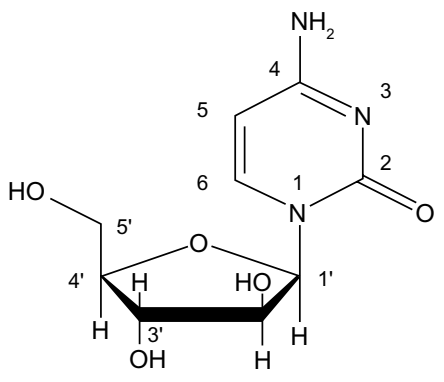

Cytarabine

Figure I Comparison of the chemical structures of decitabine (5-aza-2'-deoxycytidine) and cytarabine with that of the nucleotide deoxycytidine.

implicated in the pathogenesis of a variety of human cancers, including MDS and AML, ${ }^{32-34}$ this led to clinical interest in using low-dose decitabine in these malignancies.

\section{Phase III studies in MDS}

Two phase III studies of decitabine in the MDS population warrant mention in this review, since they included patients with $20 \%-30 \%$ bone marrow blasts who were initially classified as the refractory anemia with excess blasts in transformation subset of MDS. This group was reclassified as AML by the WHO criterion in 2002 . The first study by Kantarjian et al in 2006 randomized 170 patients with highrisk MDS to supportive care with transfusions or decitabine at a dose of $15 \mathrm{mg} / \mathrm{m}^{2}$ intravenously (IV) every 8 hours for 3 days (total dose per cycle of $135 \mathrm{mg} / \mathrm{m}^{2}$ ) every 6 weeks. ${ }^{11}$ Thirty-one of the 170 patients in this study (18\%) met the WHO criterion for AML. Patients in the decitabine arm had a $17 \%$ response rate $(9 \% \mathrm{CR})$, compared to no responses noted in the supportive-care group. An additional 13\% of patients on decitabine achieved some hematological response. Responses were durable, lasting a median of 10.3 months, and were associated with transfusion independence. Decitabine therapy was also associated with a trend towards longer time to AML progression or death (median 12.1 vs 7.8 months), but this did not reach statistical significance $(P=0.16)$. Among the patients with $>20 \%$ bone marrow blasts, three of the 17 patients randomized to the decitabine arm (19\%) achieved either a complete or partial response. Decitabine was largely well tolerated, with hematological toxicities being the most common. Based on these data, decitabine gained FDA approval for MDS.

A second randomized multicenter phase III European study published in 2011 compared decitabine to supportive care in 233 patients with MDS, including 75 patients (32\%) with $20 \%-30 \%$ bone marrow blasts. ${ }^{35}$ Decitabine was given at the same dose $\left(15 \mathrm{mg} / \mathrm{m}^{2} \mathrm{IV}\right.$ three times a day for 3 days as part of a 6-week cycle). Response rates were similar to the previous study, with $13 \%$ of patients on decitabine achieving $\mathrm{CR}, 6 \%$ achieving partial remission, and $15 \%$ achieving hematological improvement. Again, although there was a trend towards improved overall survival (median of 10.1 months versus 8.5 months, $P=0.38$ ) and AML-free survival (median of 8.8 versus 6.1 months, $P=0.24$ ), it did not reach statistical significance. However, progression-free survival (6.4 months versus 3.0 months) was significantly different $(P=0.004)$. Decitabine was also associated with improvements in patient-reported quality-of-life measures. Again, the primary toxicity remained hematological, with $25 \%$ of patients on the decitabine arm experiencing grade $3 / 4$ febrile neutropenia compared to $7 \%$ on the supportivecare arm.

\section{Phase I studies in AML}

Three phase I studies of low-dose decitabine in patients with leukemia examined safety and response to different decitabine regimens, including combination therapy with other low-intensity agents. The majority of patients in these studies were elderly, and most had relapsed AML. They set the stage for the phase II and III studies described further, and are summarized in Table 1.

The first phase I study, by Issa et al in 2004, examined four different regimens of decitabine in 50 patients with hematological malignancies, of whom nearly three-quarters carried a diagnosis of AML. ${ }^{36}$ Patients were treated with 5, 10, 15 , or $20 \mathrm{mg} / \mathrm{m}^{2}$ of decitabine daily for 10-20 days. Overall, response was noted in $32 \%$ of patients, with CR in $18 \%$. Among the AML patients, 14\% achieved CR and $8 \%$ had CR with incomplete platelet recovery (CRp). The $15 \mathrm{mg} / \mathrm{m}^{2}$ 10-day dosing was noted to be optimal, with responses observed in $65 \%$ of patients. Treatment was well tolerated, with severe elevations in liver enzymes in six patients as the primary nonhematological toxicity. 
Table I Summary of clinical trials examining low-dose decitabine in acute myeloid leukemia (AML)

\begin{tabular}{|c|c|c|c|c|c|c|}
\hline & Phase & Decitabine regimen & $\begin{array}{l}\text { Patients } \\
\text { with AML }\end{array}$ & $\begin{array}{l}\text { Median } \\
\text { age (years) }\end{array}$ & $\mathrm{CR}^{*}(\%)$ & $\begin{array}{l}\text { Median survival } \\
\text { (months) }\end{array}$ \\
\hline Issa et $\mathrm{al}^{36}$ & 1 & $\begin{array}{l}\text { Varied, with } 15 \mathrm{mg} / \mathrm{m}^{2} \text { daily for } 10 \text { days } \\
\text { noted to be optimal }\end{array}$ & 37 & 60 & 21.6 & NR \\
\hline Garcia-Manero et al ${ }^{37, * *}$ & $\mathrm{I} / \mathrm{II}$ & $15 \mathrm{mg} / \mathrm{m}^{2}$ daily for 10 days every 4 weeks & 48 & 60 & 18.8 & NR \\
\hline Blum et $\mathrm{a}^{\mid 38, * *}$ & I & $\begin{array}{l}\text { Varied, with } 20 \mathrm{mg} / \mathrm{m}^{2} \text { daily for } 10 \text { days } \\
\text { every } 4 \text { weeks noted to be optimal }\end{array}$ & 25 & 70 & 32.0 & NR \\
\hline Cashen et $\mathrm{al}^{39}$ & II & $20 \mathrm{mg} / \mathrm{m}^{2}$ daily for 5 days every 4 weeks & 55 & 74 & 25.4 & 7.7 \\
\hline Blum et $\mathrm{al}^{42}$ & II & $20 \mathrm{mg} / \mathrm{m}^{2}$ daily for 10 days every 4 weeks & 53 & 74 & 64.2 & 12.7 \\
\hline Lübbert et $\mathrm{al}^{43}$ & II & $\begin{array}{l}15 \mathrm{mg} / \mathrm{m}^{2} \text { three times daily for } 3 \text { days } \\
\text { every } 6 \text { weeks }\end{array}$ & 227 & 72 & 13.2 & 5.5 \\
\hline Kantarjian et $\mathrm{al}^{44}$ & III & $\begin{array}{l}20 \mathrm{mg} / \mathrm{m}^{2} \text { for } 5 \text { days every } 4 \text { weeks } \\
\text { vs LDAC or supportive care }\end{array}$ & $\begin{array}{l}485 \text { ( } 242 \text { with } \\
\text { decitabine) }\end{array}$ & 73 & $\begin{array}{l}\text { I } 7.8 \text { vs } 7.8 \\
(P=0.00 I)\end{array}$ & 7.7 vs $5(P=0.11)$ \\
\hline
\end{tabular}

Notes: *CR rate includes patients with complete remission with incomplete count recovery, except for Lübbert (20I0), where only CR was included. CR with incomplete count recovery in this study was combined with partial remission. **Some patients were treated with valproic acid in addition to decitabine.

Abbreviations: CR, complete remission; NR, not reported; LDAC, low-dose cytarabine.

A second phase I/II study by Garcia-Manero et al treated $54 \mathrm{AML}$ and MDS patients with a decitabine dose of $15 \mathrm{mg} / \mathrm{m}^{2} /$ day for 10 days, in combination with the histone deacetylase inhibitor valproic acid. ${ }^{37}$ Valproic acid was escalated to $50 \mathrm{mg} / \mathrm{kg} / \mathrm{day}$, with an acceptable level of neurotoxicity. The majority of the patients had AML (48 of 54 ), with a median age of 60 years. Overall, $22 \%$ of patients had a response, including ten with CR and two with CRp. Notably, $50 \%$ of the previously untreated AML patients had a response. Overall survival was 6 months, but median survival was as high as 15.3 months in the responders. The primary nonhematological toxicity was anorexia and confusion, with the latter primarily attributed to the valproic acid.

A third phase I study by Blum et al also examined decitabine in combination with valproic acid in AML patients. ${ }^{38}$ Twenty-five patients (twelve untreated, 13 relapsed) with AML with a median age of 70 years were enrolled. Two doses of decitabine were administered $\left(15 \mathrm{mg} / \mathrm{m}^{2} /\right.$ day and $20 \mathrm{mg} / \mathrm{m}^{2} /$ day) for 10 days, and the higher dose was defined as the optimum biological dose, as it induced higher rates of reexpression of $E R$ and $P 15$ (genes methylated in AML). Three different valproic acid dosing regimens were then combined with the $20 \mathrm{mg} / \mathrm{m}^{2}$ dose of decitabine. Confusion again was the primary toxicity observed with valproic acid, especially with doses above $20 \mathrm{mg} / \mathrm{kg} /$ day. The overall response rate was $44 \%$ in the intention-to-treat group and $52 \%$ (eleven of 21) in assessable patients. The addition of valproic acid did not appear to confer any additional benefit. The authors concluded that given valproic acid's narrow therapeutic index and toxicity, decitabine alone or in combination with alternative histone-deacetylating agents should be the focus of future studies.

\section{Phase II studies in AML}

Three phase II studies established decitabine's activity in elderly AML patients. In a multicenter study, Cashen et al treated 55 newly diagnosed older AML patients with intermediate- or poor-risk cytogenetics with decitabine $20 \mathrm{mg} / \mathrm{m}^{2} /$ day for 5 days, repeated on a 4 -week cycle. ${ }^{39}$ The 5 -day decitabine regimen was drawn from studies that had found this outpatient schedule to be effective and well tolerated in MDS patients. ${ }^{40,41}$ The study population was a highrisk group, with a median age of 74 years, $42 \%$ secondary AML, and median baseline bone marrow blast percentage of $50 \%$. After a median of three cycles of decitabine, the overall response rate was $25 \%$ and an additional $29 \%$ had stable disease. The majority of the responders achieved morphological CR (13 of 14), with one patient achieving CR with incomplete count recovery (CRi). Response rates were preserved in patients with poor-risk cytogenetics, a subgroup that has historically derived limited benefit with other agents such as LDAC. ${ }^{19}$ While a majority of the study population did not have proliferative AML (median white blood cell count 2.7), it was notable that only one (7\%) of 14 patients with a peripheral blast count over $1,000 / \mu \mathrm{L}$ achieved CR. Among patients with a cytogenetic abnormality at baseline, five of 25 achieved cytogenetic remission. The time to achieve CR was 4.5 cycles, favoring differentiation rather than cytotoxicity as the primary mechanism of action and indicating that persistence with the medication is needed if the drug is tolerated and the disease is stable. Median survival was 7.7 months for the full cohort, but was 14 months for the responders. The drug was fairly well tolerated, with myelosuppression, febrile neutropenia (29\%), and fatigue as the major toxicities and a 30-day mortality of $7 \%$. This promising response rate and toxicity profile set the 
stage for the use of this decitabine dose in the phase III study described later.

A single-center phase II trial by Blum et al increased the first-cycle dose of decitabine to $20 \mathrm{mg} / \mathrm{m}^{2} /$ day for 10 days, with cycle length reduced to 5 days if response was observed. ${ }^{42}$ Fifty-three older patients with newly diagnosed AML were treated. In contrast to the Cashen et al study, ${ }^{39}$ this study population included patients with good-risk prognostic factors (19\% of enrollees). The median age was 74 years, and $36 \%$ of patients had secondary AML. After treatment with a median of four cycles of decitabine, the response rate was 64\% (34 of 53), with 49\% CR and 15\% CRi. The response rate was similarly high across all cytogenetic subgroups, and was even observed in patients with high circulating blasts, where the response was $50 \%$ for patients with a white blood cell count $>50,000$. Interestingly, in eleven patients with monosomy 7 or deletion of $7 q$, the response rate was remarkable - 91\%. Median survival was 12.7 months, and disease-free survival for patients in CR was 10.6 months. The primary toxicity remained myelosuppression. The higher dose of decitabine (10-day cycle), along with longer exposure to the drug (four cycles), resulted in higher rates of febrile neutropenia compared to prior studies (68\%), but overall decitabine was well tolerated, with only one death $(2 \%)$ within 30 days of therapy. The authors correlated response with pretreatment levels of microRNA29b (miR29b), an miRNA that specifically inhibits DNA methyltransferase expression. Patients who responded to decitabine tended to have higher levels of miR $29 \mathrm{~b}(P=0.02)$, indicating that it could potentially be used as a predictive marker for response.

A third large phase II study, by Lübbert et al, treated 227 elderly AML patients at nine centers in Europe, with an MDS dosing schedule of decitabine at $15 \mathrm{mg} / \mathrm{m}^{2}$ every 8 hours for 3 days as part of a 6-week cycle. ${ }^{43}$ Patients who had stable disease or antileukemic effect after cycle 1 were given alltrans retinoic acid (ATRA) at the next cycle, to determine if hypomethylation promoted ATRA susceptibility even in the absence of $P M L-R A R A$ fusion (based on preclinical studies). The study population had poor prognostic indicators, with a median age of 72 years, $51 \%$ secondary AML, $32 \%$ adverse cytogenetics, and median bone marrow blasts of $56 \%$. After receiving a median of two cycles, objective response was achieved in $26 \%$ (59 patients), with CR in 30 patients ( $13 \%$ ) and partial remission ( $5 \%-25 \%$ bone marrow blasts or CRi) in 29 additional participants. A further $26.4 \%$ of patients responded with "antileukemic effect," defined as $25 \%$ reduction in blasts without meeting the criterion for partial remission, and another $25 \%$ had stable disease. Response rates were similar in patients with adverse cytogenetics (29\%) and those with the very poor-risk monosomal karyotype (37\%). Median survival was 5.5 months. The inclusion of ATRA with cycle 2 did not prolong survival in patients. Decitabine was again well tolerated, with $33.5 \%$ of patients experiencing grade 3 or 4 toxicity. Mortality after the first cycle was $12.8 \%$. While the CR rate in this study was lower than previous ones, at $13 \%$, this may be reflective of the study design, which called for application of four courses of therapy and then optional maintenance therapy without systematic recording of improvement in response rate while on maintenance. The authors concluded that decitabine was well tolerated, with encouraging responses noted in patients with poor-risk cytogenetics.

\section{Phase III study in AML}

The phase III randomized trial examining outcomes with decitabine in elderly AML patients was an open-label study of 485 patients in 15 countries randomized in a $1: 1$ fashion to decitabine or their treatment choice (TC) of either LDAC or supportive care. ${ }^{44}$ The majority of the patients in the TC arm received LDAC therapy (218 of the 243 patients) at a dose of $20 \mathrm{mg} / \mathrm{m}^{2}$ daily subcutaneously for 10 days as a 4-week cycle. Notably, the daily LDAC dosing was lower than prior studies, including those by Burnett et al (which used a twice-daily regimen), ${ }^{19}$ with the reduction done for dosing convenience. The decitabine dose was the same as that used in the phase II study by Cashen et al, ${ }^{39}$ at $20 \mathrm{mg} / \mathrm{m}^{2} /$ day IV for 5 days as a 4-week cycle. The population was high-risk, with an average age of 73 years, median bone marrow blast count of $46 \%$, and $35.3 \%$ with secondary AML. Patients with favorable cytogenetics were excluded. The primary end point was overall survival, which was prospectively specified to occur at 385 deaths $(80 \%)$, and the study was powered to detect a $25 \%$ reduction in mortality. Secondary end points included the combination of CR and CRp and safety.

While there was a trend towards improved survival with decitabine (7.7- versus 5.0-month median survival) at the prospectively defined time point, the hazard ratio of 0.85 was not statistically significant $(P=0.108)$. However, statistical significance was achieved in a follow-up ad hoc analysis performed 1 year later, when $92 \%$ of deaths had occurred. At that time point, the median survival remained the same, and the hazard ratio was $0.82(P=0.037)$. Also, by censoring patients who received subsequent antileukemic therapy $(37.6 \%$ patients in the decitabine arm and $44.4 \%$ in the TC arm), 
overall survival was again significant (8.5- versus 5.3-month median survival, $P=0.044$ ).

Multivariate analysis of survival demonstrated decitabine superiority in patients over 75 years old, those with an Eastern Cooperative Oncology Group performance status of 2 , and those with over $30 \%$ marrow blasts. Interestingly, no difference between decitabine and LDAC was noted in the subgroup with $20 \%-30 \%$ marrow blasts. This group did derive benefit from decitabine in the phase III studies of decitabine in MDS, ${ }^{11,34,35}$ but the control group in those studies was supportive care alone, which may account for the discrepancy in results.

Regarding the secondary end points, patients on decitabine had significantly improved rates of CR and CRp $(17.8 \%$ vs $7.8 \%$ in the TC group) at the prespecified cutoff, with an odds ratio of $2.5(1.4-4.8, P=0.001)$. The safety profile of decitabine was similar to LDAC, with the majority of patients in both arms experiencing some grade 3 or 4 toxicity while taking the study drug. The primary toxicity was hematologic, with a higher incidence of thrombocytopenia (40\% vs 32\%), anemia (34\% vs 25\%), and neutropenia (32\% vs $18 \%$ ) in the decitabine arm. Infectious complications were also slightly higher in the decitabine group, with febrile neutropenia ( $32 \%$ vs $22 \%$ ) and pneumonia ( $21 \%$ vs $18 \%$ ) being the two most common. However, the authors noted that patients in the decitabine arm were exposed to the study medication longer than the LDAC group (4.4 months vs 2.4 months), which likely contributed to greater reporting of adverse events. Discontinuation of the study drug was similar for decitabine $(6 \%)$ and cytarabine $(8 \%)$. The authors concluded that decitabine was at least as well tolerated as LDAC, with no major differences in safety.

While this phase III study demonstrated decitabine to be an effective alternative to LDAC for high-risk elderly patients with AML, its results have been fraught with controversy. Based on the same trial data, the FDA Oncological Drug Advisory Committee (ODAC) voted against approving decitabine for the indication of AML in elderly patients, but the European Medicines Agency Committee for Medicinal Products for Human Use approved it. The controversy stems largely from difficulty in assigning superiority to decitabine when the survival differences at the prospectively specified end point did not reach significance. While mature data with more deaths included did show significantly improved survival with decitabine, the fact that it was an unplanned analysis led to concern for a false-positive result. ${ }^{45}$ The FDA panel also noted that the CR rate with LDAC (8.4\%) was lower than prior studies that had reported 15\%-20\%. ${ }^{13,19,44,45,46}$
While comparisons across trials are fraught with bias due to differences in patient characteristics, the fact that a lower dose of cytarabine was used does raise the possibility that suboptimal cytarabine dosing in the control arm contributed to this lower CR rate. Finally the ODAC panel also expressed concern that in the Western European study population (where LDAC is more frequently used), the survival trend favored LDAC versus decitabine (median survival 12.5 versus 9 months, $P=0.91) .{ }^{45}$ However the authors refuted the merits of this argument by noting that the patients in the decitabine arm in Europe had worse baseline characteristics and many patients in the TC arm in Europe subsequently received further therapy with hypomethylating agents, making it difficult to derive any conclusions from this subgroup analysis. ${ }^{47}$

Prior studies have suggested that certain subgroups of elderly AML patients benefit more from hypomethylating agents. The phase II data by Cashen et $\mathrm{al}^{39}$ and data from azacitidine ${ }^{46}$ have suggested that hypomethylating agents may be particularly beneficial in hypoproliferative AML. However, the data from the phase III study did not analyze peripheral blast count as an independent variable. Additional studies have suggested that decitabine may also confer greater benefit to subgroups with specific molecular characteristics. Monosomal karyotype patients, who have worse outcomes with standard cytotoxic chemotherapy, ${ }^{48}$ had impressive response rates to decitabine in phase II AML studies ${ }^{42,43}$ and to azacitidine in MDS. ${ }^{49,50}$ However, outcome based on karyotype was not provided in the phase III study either, and could be a subject of further investigation.

\section{Decitabine as a bridge to transplant in AML patients}

A single-center study has explored decitabine as a bridge to reduced-intensity allogeneic transplant for elderly patients with a good performance status. ${ }^{51,52}$ Traditionally, standard induction chemotherapy has been used to achieve CR prior to transplant. Lübbert et al tested decitabine instead, with the hypothesis that the drug's milder toxicity would reduce up-front mortality and may in fact enhance the graftversus-leukemia effect by upregulating human leukocyteantigen expression. ${ }^{51}$ They reported their experience with 15 consecutive elderly patients (nine with AML and six with MDS), with a median age of 69 years, treated with decitabine at $15 \mathrm{mg} / \mathrm{m}^{2}$ every 8 hours for 3 days as part of a 6-week cycle. After a median of five cycles of decitabine, five patients achieved CR. Allografting was performed after conditioning with a reduced-intensity regimen of fludarabine, 
carmustine, and melphalan. The median blast percentage for study population was $24 \%$ both at the start of induction and at the time of transplant. After transplant, one patient died of infection before engraftment, but eight of ten patients for whom data were available had full donor chimerism at 30 and 100 days after transplant. The rates of graft-versus-host disease were not higher than expected. While median duration of CR after transplant was only 5 months, survival at 1 year was $47 \%$, at 2 years was $30 \%$, and three patients continued to be alive over 5 years after their transplant at the time of publication of their update. ${ }^{52}$ While this was only a small single-center study, it supports further investigation of decitabine induction chemotherapy as a bridge to transplant.

\section{Conclusion and future directions}

The hypomethylating agent decitabine at low doses promotes cellular differentiation by reversing epigenetic suppression of genes, and has shown efficacy in producing remissions lasting several months in a subset of elderly patients with MDS and AML. It can be given as outpatient therapy daily for 5-10 days per cycle. It is relatively well tolerated in the elderly population, with myelosuppression and febrile neutropenia as the primary toxicity. Multiple cycles of decitabine may be needed to achieve remission, and therefore treatment should be continued as long as the disease is stable. A recent phase III study demonstrated higher rates of complete remission with decitabine therapy compared to low-dose cytarabine in elderly AML patients, but the survival difference failed to reach significance. ${ }^{44,45}$

At present, the role of decitabine in the treatment of elderly AML patients is yet to be fully defined. At this time, there are multiple ongoing studies to further delineate decitabine's efficacy in elderly AML patients. ${ }^{53}$ Given its relatively mild toxicity, decitabine continues to be an attractive agent for combination therapy with other antileukemic agents. Phase I and II studies examining decitabine in combination with low-dose cytarabine (NCT01829503), bortezomib (NCT01420926), plerixafor (NCT01352650), and the tyrosine-kinase inhibitors midostaurin (NCT01846624) and sorafenib (NCT01861314) are all currently under way. ${ }^{53}$ There continues to be interest in combining decitabine with newer, less toxic histone deacetylase inhibitors, such as AR-42 (NCT01798901), with the rationale that promoting histone acetylation would lead to an open chromatin configuration and permit repressed gene expression synergistically, as has been noted in preclinical studies. ${ }^{54}$ Correlative studies are also ongoing to define cytogenetic and molecular characteristics that predict response to decitabine (NCT01687400).
A retrospective analysis of $D N M T 3 A$ mutation status and decitabine response demonstrated a response rate of $75 \%$ in a cohort of eight patients, but this needs further validation in larger studies. ${ }^{55}$ An observation from the phase II data that monosomal karyotype elderly AML patients may have a disproportionate benefit from decitabine also warrants further attention. An oral formulation of decitabine is also under investigation, ${ }^{56}$ and may provide a more convenient option for elderly patients. Another trial comparing a 10-day versus 5-day decitabine regimen is also under way, given the higher response rates in the single-center phase II study with the 10-day regimen (NCT01786343). Decitabine may also have a role as maintenance therapy or as a bridge to allogeneic stem cell transplant. ${ }^{51,52}$

In conclusion, elderly AML patients with intermediateand poor-risk cytogenetics continue to have a very poor prognosis. Standard induction chemotherapy has a high up-front mortality in this population, with a high likelihood of relapse even if remission is achieved. Decitabine is a welltolerated therapeutic alternative, and recent clinical evidence has shown it to be effective in producing remissions lasting several months or disease stabilization in a subset of this population. Further studies are needed to define which subset of elderly patients is most likely to derive maximal benefit from single-agent decitabine therapy.

\section{Disclosure}

The authors report no conflicts of interest in this work.

\section{References}

1. National Cancer Institute. SEER stat fact sheets: acute myeloid leukemia. Available from: http://seer.cancer.gov/statfacts/html/amyl.html Accessed June 16, 2013.

2. Medeiros BC, Othus M, Fang M, Roulston D, Appelbaum FR. Prognostic impact of monosomal karyotype in young adult and elderly acute myeloid leukemia: the Southwest Oncology Group (SWOG) experience. Blood. 2010;116(13):2224-2228.

3. Grimwade D, Walker H, Harrison G, et al. The predictive value of hierarchical cytogenetic classification in older adults with acute myeloid leukemia (AML): analysis of 1065 patients entered into the United Kingdom Medical Research Council AML11 trial. Blood. 2001;98(5): 1312-1320.

4. Bacher U, Kern W, Schnittger S, Hiddemann W, Haferlach T, Schoch C. Population-based age-specific incidences of cytogenetic subgroups of acute myeloid leukemia. Haematologica. 2005;90(11):1502-1510.

5. Schneider F, Hoster E, Schneider S, et al. Age-dependent frequencies of NPM1 mutations and FLT3-ITD in patients with normal karyotype AML (NK-AML). Ann Hematol. 2012;91(1):9-18.

6. Leith CP, Kopecky KJ, Godwin J, et al. Acute myeloid leukemia in the elderly: assessment of multidrug resistance (MDR1) and cytogenetics distinguishes biologic subgroups with remarkably distinct responses to standard chemotherapy. A Southwest Oncology Group study. Blood. 1997;89(9):3323-3329.

7. Stresemann C, Lyko F. Modes of action of the DNA methyltransferase inhibitors azacytidine and decitabine. Int J Cancer. 2008;123(1):8-13. 
8. Christman JK. 5-Azacytidine and 5-aza-2'-deoxycytidine as inhibitors of DNA methylation: mechanistic studies and their implications for cancer therapy. Oncogene. 2002;21(35):5483-5495.

9. Silverman LR, Demakos EP, Peterson BL, et al. Randomized controlled trial of azacitidine in patients with the myelodysplastic syndrome: a study of the cancer and leukemia group B. J Clin Oncol. 2002;20(10):2429-2440.

10. Fenaux P, Mufti GJ, Hellstrom-Lindberg E, et al. Efficacy of azacitidine compared with that of conventional care regimens in the treatment of higher-risk myelodysplastic syndromes: a randomised, open-label, phase III study. Lancet Oncol. 2009;10(3):223-232.

11. Kantarjian H, Issa JP, Rosenfeld CS, et al. Decitabine improves patient outcomes in myelodysplastic syndromes: results of a phase III randomized study. Cancer. 2006;106(8):1794-1803.

12. Löwenberg B, Ossenkoppele GJ, van Putten W, et al. High-dose daunorubicin in older patients with acute myeloid leukemia. $N$ Engl J Med. 2009;361(13):1235-1248.

13. Tilly H, Castaigne S, Bordessoule D, et al. Low-dose cytarabine versus intensive chemotherapy in the treatment of acute nonlymphocytic leukemia in the elderly. J Clin Oncol. 1990;8(2):272-279.

14. Kantarjian H, O'Brien S, Cortes J, et al. Results of intensive chemotherapy in 998 patients age 65 years or older with acute myeloid leukemia or high-risk myelodysplastic syndrome: predictive prognostic models for outcome. Cancer. 2006;106(5):1090-1098.

15. Oran B, Weisdorf DJ. Survival for older patients with acute myeloid leukemia: a population-based study. Haematologica. 2012;97(12): 1916-1924.

16. Rowe JM, Neuberg D, Friedenberg W, et al. A phase 3 study of three induction regimens and of priming with GM-CSF in older adults with acute myeloid leukemia: a trial by the Eastern Cooperative Oncology Group. Blood. 2004;103(2):479-485.

17. Estey E, de Lima M, Tibes R, et al. Prospective feasibility analysis of reduced-intensity conditioning (RIC) regimens for hematopoietic stem cell transplantation (HSCT) in elderly patients with acute myeloid leukemia (AML) and high-risk myelodysplastic syndrome (MDS). Blood. 2007;109(4):1395-1400.

18. Menzin J, Lang K, Earle CC, Kerney D, Mallick R. The outcomes and costs of acute myeloid leukemia among the elderly. Arch Inter Med. 2002;162(14):1597-1603.

19. Burnett AK, Milligan D, Prentice AG, et al. A comparison of lowdose cytarabine and hydroxyurea with or without all-trans retinoic acid for acute myeloid leukemia and high-risk myelodysplastic syndrome in patients not considered fit for intensive treatment. Cancer. 2007;109(6):1114-1124.

20. Castaigne $\mathrm{S}$, Pautas $\mathrm{C}$, Terre $\mathrm{C}$, et al. Effect of gemtuzumab ozogamicin on survival of adult patients with de-novo acute myeloid leukaemia (ALFA-0701): a randomised, open-label, phase 3 study. Lancet. 2012;379(9825):1508-1516.

21. Petersdorf SH, Kopecky KJ, Slovak M, et al. A phase 3 study of gemtuzumab ozogamicin during induction and postconsolidation therapy in younger patients with acute myeloid leukemia. Blood. 2013;121(24):4854-4860.

22. National Comprehensive Cancer Network. NCCN clinical practice guidelines in oncology version 2.2013: acute myeloid leukemia. Available from: http://www.nccn.org/professionals/physician_gls/pdf/ aml.pdf. Accessed June 16, 2013.

23. Tran H, Yang D. Clofarabine in the treatment of newly diagnosed acute myeloid leukemia in older adults. Ann Pharmacother. 2012;46(1): 89-96.

24. Keating GM. Azacitidine: a review of its use in the management of myelodysplastic syndromes/acute myeloid leukaemia. Drugs. 2012;72(8):1111-1136.

25. Momparler RL, Momparler LF, Samson J. Comparison of the antileukemic activity of 5-AZA-2'-deoxycytidine, 1-beta-Darabinofuranosylcytosine and 5-azacytidine against L1210 leukemia. Leuk Res. 1984;8(6):1043-1049.
26. Petti MC, Mandelli F, Zagonel V, et al. Pilot study of 5-aza-2'deoxycytidine (decitabine) in the treatment of poor prognosis acute myelogenous leukemia patients: preliminary results. Leukemia. 1993; 7 Suppl 1:36-41.

27. Willemze R, Suciu S, Archimbaud E, et al. A randomized phase II study on the effects of 5-Aza-2'-deoxycytidine combined with either amsacrine or idarubicin in patients with relapsed acute leukemia: an EORTC Leukemia Cooperative Group phase II study (06893). Leukemia. 1997;11 Suppl 1:S24-S27.

28. Jones PA, Taylor SM. Cellular differentiation, cytidine analogs and DNA methylation. Cell. 1980;20(1):85-93.

29. Momparler RL. Pharmacology of 5-Aza-2'-deoxycytidine (decitabine). Semin Hematol. 2005;42(3 Suppl 2):S9-S16.

30. Baylin SB. DNA methylation and gene silencing in cancer. Nat Clin Pract Oncology. 2005;2 Suppl 1:S4-S11.

31. Herman JG, Baylin SB. Gene silencing in cancer in association with promoter hypermethylation. N Engl J Med. 2003;349(21):2042-2054.

32. Leone G, Teofili L, Voso MT, Lübbert M. DNA methylation and demethylating drugs in myelodysplastic syndromes and secondary leukemias. Haematologica. 2002;87(12):1324-1341.

33. Schoofs T, Müller-Tidow C. DNA methylation as a pathogenic event and as a therapeutic target in AML. Cancer Treat Rev. 2011;37 Suppl 1: S13-S18.

34. Agrawal S, Hofmann WK, Tidow N, et al. The C/EBPdelta tumor suppressor is silenced by hypermethylation in acute myeloid leukemia. Blood. 2007;109(9):3895-3905.

35. Lübbert M, Suciu S, Baila L, et al. Low-dose decitabine versus best supportive care in elderly patients with intermediate- or high-risk myelodysplastic syndrome (MDS) ineligible for intensive chemotherapy: final results of the randomized phase III study of the European Organisation for Research and Treatment of Cancer Leukemia Group and the German MDS Study Group. J Clin Oncol. 2011;29(15):1987-1996.

36. Issa JP, Garcia-Manero G, Giles FJ, et al. Phase 1 study of low-dose prolonged exposure schedules of the hypomethylating agent 5-aza2 '-deoxycytidine (decitabine) in hematopoietic malignancies. Blood. 2004;103(5):1635-1640.

37. Garcia-Manero G, Kantarjian HM, Sanchez-Gonzalez B, et al. Phase 1/2 study of the combination of 5-aza-2'-deoxycytidine with valproic acid in patients with leukemia. Blood. 2006;108(10):3271-3279.

38. Blum W, Klisovic RB, Hackanson B, et al. Phase I study of decitabine alone or in combination with valproic acid in acute myeloid leukemia. J Clin Oncol. 2007;25(25):3884-3891.

39. Cashen AF, Schiller GJ, O’Donnell MR, DiPersio JF. Multicenter, phase II study of decitabine for the first-line treatment of older patients with acute myeloid leukemia. J Clin Oncol. 2010;28(4):556-561.

40. Kantarjian H, Oki Y, Garcia-Manero G, et al. Results of a randomized study of 3 schedules of low-dose decitabine in higher-risk myelodysplastic syndrome and chronic myelomonocytic leukemia. Blood. 2007;109(1):52-57.

41. Steensma DP, Baer MR, Slack JL, et al. Multicenter study of decitabine administered daily for 5 days every 4 weeks to adults with myelodysplastic syndromes: the alternative dosing for outpatient treatment (ADOPT) trial. J Clin Oncol. 2009;27(23):3842-3848.

42. Blum W, Garzon R, Klisovic RB, et al. Clinical response and miR-29b predictive significance in older AML patients treated with a 10-day schedule of decitabine. Proc Natl Acad Sci U S A. 2010;107(16): 7473-7478.

43. Lübbert M, Rüter BH, Claus R, et al. A multicenter phase II trial of decitabine as first-line treatment for older patients with acute myeloid leukemia judged unfit for induction chemotherapy. Haematologica. 2012;97(3):393-401.

44. Kantarjian HM, Thomas XG, Dmoszynska A, et al. Multicenter, randomized, open-label, phase III trial of decitabine versus patient choice, with physician advice, of either supportive care or low-dose cytarabine for the treatment of older patients with newly diagnosed acute myeloid leukemia. J Clin Oncol. 2012;30(21):2670-2677. 
45. Sekeres MA, Steensma DP. Boulevard of broken dreams: drug approval for older adults with acute myeloid leukemia. J Clin Oncol. 2012;30(33): 4061-4063.

46. Fenaux P, Mufti GJ, Hellstrom-Lindberg E, et al. Azacitidine prolongs overall survival compared with conventional care regimens in elderly patients with low bone marrow blast count acute myeloid leukemia. J Clin Oncol. 2010;28(4):562-569.

47. Kantarjian H, Wilson W, Ravandi F, Estey E. Decitabine in older adults with acute myeloid leukemia: why was the dream broken? J Clin Oncol. 2013;31(14):1795-1796.

48. Ravandi F, Issa JP, Garcia-Manero G, et al. Superior outcome with hypomethylating therapy in patients with acute myeloid leukemia and high-risk myelodysplastic syndrome and chromosome 5 and 7 abnormalities. Cancer. 2009;115(24):5746-5751.

49. Furlan I, Batz C, Flotho C, et al. Intriguing response to azacitidine in a patient with juvenile myelomonocytic leukemia and monosomy 7 . Blood. 2009;113(12):2867-2868.

50. Rüter B, Wijermans P, Claus R, Kunzmann R, Lübbert M. Preferential cytogenetic response to continuous intravenous low-dose decitabine (DAC) administration in myelodysplastic syndrome with monosomy 7. Blood. 2007;110(3):1080-1082; author reply 1083.
51. Lübbert M, Bertz H, Rüter B, et al. Non-intensive treatment with lowdose 5-aza-2'-deoxycytidine (DAC) prior to allogeneic blood SCT of older MDS/AML patients. Bone Marrow Transplant. 2009;44(9): 585-588.

52. Lübbert M, Bertz H, Müller MJ, Finke J. When azanucleoside treatment can be curative: nonintensive bridging strategy before allografting in older patients with myelodysplastic syndrome/acute myeloid leukemia. J Clin Oncol. 2013;31(6):822-823.

53. ClinicalTrials.gov [website on the Internet]. Available from: http:// www.clinicaltrials.gov. Accessed June 16, 2013.

54. Kalac M, Scotto L, Marchi E, et al. HDAC inhibitors and decitabine are highly synergistic and associated with unique gene-expression and epigenetic profiles in models of DLBCL. Blood. 2011;118(20): 5506-5516.

55. Metzeler KH, Walker A, Geyer S, et al. DNMT3A mutations and response to the hypomethylating agent decitabine in acute myeloid leukemia. Leukemia. 2012;26(5):1106-1107.

56. Lavelle D, Vaitkus K, Ling Y, et al. Effects of tetrahydrouridine on pharmacokinetics and pharmacodynamics of oral decitabine. Blood. 2012;119(5):1240-1247.
Cancer Management and Research

\section{Publish your work in this journal}

Cancer Management and Research is an international, peer-reviewed open access journal focusing on cancer research and the optimal use of preventative and integrated treatment interventions to achieve improved outcomes, enhanced survival and quality of life for the cancer patient The journal welcomes original research, clinical \& epidemiological

\section{Dovepress}

studies, reviews \& evaluations, guidelines, expert opinion \& commentary, case reports \& extended reports. The manuscript management system is completely online and includes a very quick and fair peerreview system, which is all easy to use. Visit http://www.dovepress.com/ testimonials.php to read real quotes from published authors. 